\title{
Lubrication Properties of Hydrogel-Coated Polyethylene Head
}

\author{
Yasunari Ishikawa ${ }^{1}$ and Tadashi Sasada \\ ${ }^{1}$ Chiba Institute of Technology, Mechanical Science, Narashino 275-8588, Japan
}

Frictional behavior of stainless steel cup against polyethylene head coated with dimethylacrylamide (DMAA) hydrogel was studied using a pendulum type friction tester. The friction coefficient of the pairs lubricated with Hyaluronic acid aq (HA) was as about 0.01, which is similar to that of living human hip or knee joint. The friction of the pair increased with loading time. When the coated head was immersed in the HA lubricant during an interval of repeated swing motions of the pendulum, the friction coefficient was above low value. The friction coefficient of the pair lubricated with a hydrophobic liquid (silicone oil) was as a high value of 0.4 , close to the value without any lubricant. The friction coefficients of pairs lubricated with HA were not dependent on the load. The coefficients of friction lubricated with the biological factor (albumin, phospholipid) additive HA showed a little increase. Especially with albumin, the coefficient of friction increased with load. This behavior may be caused by the attachment albumin to hydrophilic high molecular chain by chemical adsorption. From these experimental observations, it is surmised that the low friction shown in coated PE head is caused by the formation of highly viscous zone through hydration of DMAA at the solid/liquid interface.

(Received October 20, 2003; Accepted January 9, 2004)

Keywords: low frictional coefficient, artificial joint, hydrogel, grafted polymerization, surface modification

\section{Introduction}

The lubrication mechanism at low frictional area of the organism has not been clarified yet. Therefore, it is important to understand the low frictional lubrication mechanism of the organism cartilage surface for the lubrication property improvement of the future artificial joint. It is generally considered that the lubrication mechanism of the joint manifests low friction, since hydrodynamic lubrication is promoted by hydrous property of the cartilage. ${ }^{1-3)}$ However, it is difficult to consider the low friction by hydrodynamic lubrication in the condition (long-time standing condition) that a fluid film does not seem to exist in the cartilage surface.

Mabuchi et al. ${ }^{4)}$ reported that, when the friction coefficient rises, the gelled material on the cartilage surface is wiped. Even if joint synovia is administered, the friction coefficient does not lower. From this fact, we assumed that the gelled material was contributing for the lowering of the friction.

In our earlier reports, ${ }^{5)}$ we reported the gel on the cartilage surface is schematically shown in Fig. 1. Collagen network exists so that the articular cartilages cover the epiphysis. Hyaluronic acid is extended from the collagen network.

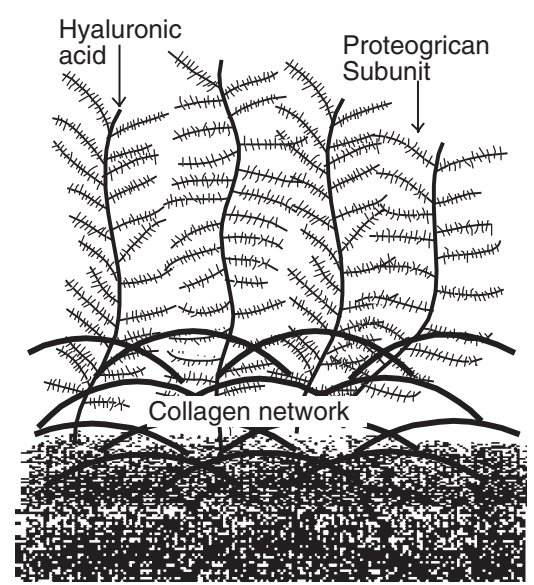

Fig. 1 Schematic representation of hydrogel on cartilage. ${ }^{5)}$
Proteoglycan aggregate is extended like the foliage from the hyaluronic acid. We considered that the articular cartilage surface had been gelled by the hydration of hydrophilic proteoglycan aggregate in the joint synovia.

In this study, we assumed that the hydrogel (hydrate of the hydrophilic macromolecule chain) was a cause of low friction. Then, we evaluated the frictional property of hydrogel coated PE head (polyethylene head that hydrophilic polymer chain was chemically covered) by using the pendulum type friction tester.

Furthermore, we examined the load properties of hydrogel coated PE head and the frictional property of the hydrogel in adding biological factor to hyaluronic acid by using the pendulum type friction tester.

\section{Method}

\subsection{Sample}

The SUS316 of diameter of $\phi 38 \mathrm{~mm}$ was used for the cup, and high density polyethylene (PE) of diameter of $\phi 37.8 \mathrm{~mm}$ was used for the head. The diameter clearance was made to be $0.2 \mathrm{~mm}$. A part of PE head was coated by benzophenone as a sensitizer. The photografting on the sensitizer-coated PE head was carried out using high pressure mercury lamp in $10 \mathrm{wt} \%$ Dimethylacrylamide monomer solution. ${ }^{6,7)}$ Frictional surface was coated with hydrogel layer (hydrophilic polymer chain) by this method. Thickness of gel layer is about $10 \mu \mathrm{m} .^{8)}$

\subsection{Lubricant}

Lubricants were $1 \%$ hyaluronic acid (HA) aqueous solution (molecular weight: 900000, pH: 6.67), $10 \mathrm{wt} \%$ glycerin aqueous solution, distilled water, siloxane $(0.4 \mathrm{Pc})$ and DMAA homopolymer. The DMAA homopolymer is made by the graft polymerization on the surface of polyethylene head. The DMAA homopolymer was used in order to examine how combination/non-binding of the hydrophilic macromolecule chain affected the friction. And, Siloxane was used in order to examine the effect of the dehydration of 


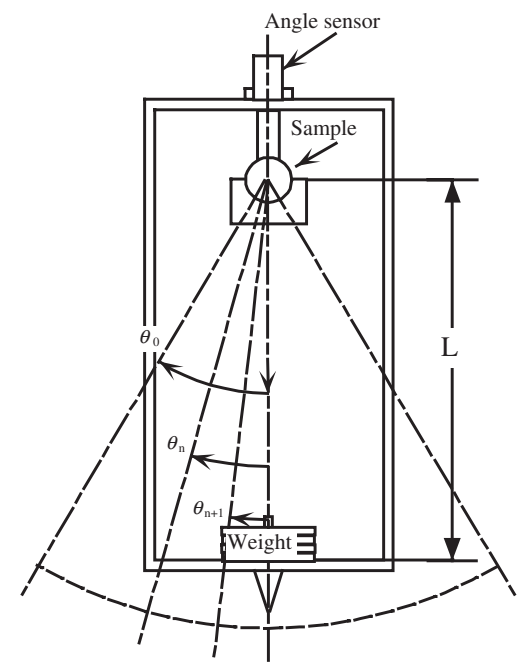

Fig. 2 Schematic illustration of pendulum tester. L: Effective arm length of pendulum.

the hydrophilic macromolecule chain on the friction.

Furthermore, along with Hyaluronic acid lubricant, 3 wt $\%$ Albumins, and $1 \mathrm{wt} \%$ Phospholipid were added.

\subsection{The measurement method of the friction}

Figure 2 shows schematic drawings of the pendulum type friction tester, which is a method generally using for the frictional measurement of joints. It was assumed that the potential energy of pendulum center of gravity was spent in the friction heat energy with the sliding of friction surfaces, and the friction coefficient was obtained from the extinction curve of deflection angle as following.

$$
f=(l / 4 r) * \Delta \theta
$$

( $f$ : friction coefficient, $L$ : effective arm length of pendulum, $r$ : friction surface radius. $\Delta \theta$ : The attenuation angle of an every vibration. (rad)

(The initial amplitude: $0.1 \mathrm{rad}$, Load: $10 \mathrm{~kg}$, effective arm length of pendulum: $470 \mathrm{~mm}$ )

The amplitude attenuation of the pendulum was measured by the angle sensor, and recorded by pen recorder. In order to obtain the hydrodynamic lubrication from the initial stage, cup and sphere were tilted to the no contacting condition in 0.1 degrees. Subsequently the pendulum was made to oscillate from the above condition, in which a lubricating film between sphere and cup had not broken down.

In order to examine water content effect of the gel layer on the friction, the frictional coefficient of hydrogel was measured under continuous or intermittent load.

In case of continuous load condition, specimen was never lifted up during measurement. However, in case of intermittent load condition, polyethylene head was lifted every 1 measurement, the friction coefficient was measured after the gel layer fully contained water.

\section{Results}

\subsection{Lubrication properties of the hydrogel}

Figure 3 shows extinction curves in rubbing untreated PE

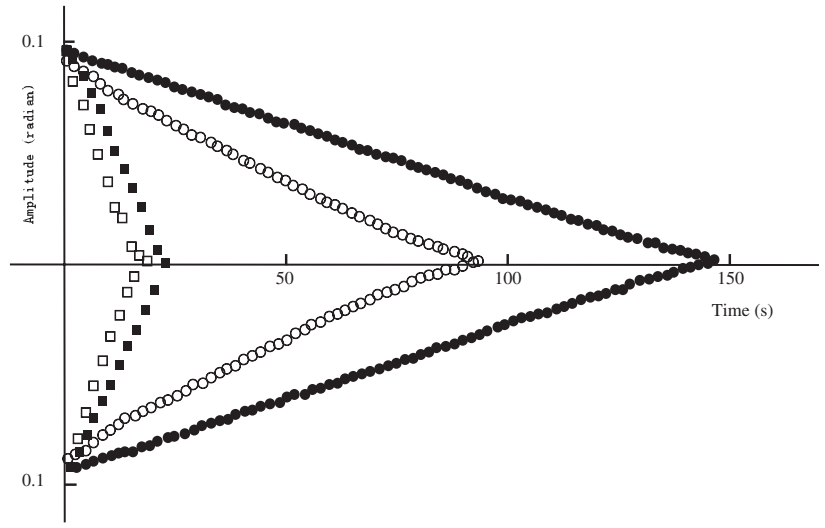

Fig. 3 Damping pattern of pendulum oscillation. Lubricants were glycerin (open) and hyaluronic acid (close). Square: PE. Circle: hydrogel-coated PE.

vs. SUS316 and the hydrogel coated PE vs. SUS316. The swing of pendulum immediately stopped, since the friction coefficient of untreated PE was high. But, Hydrogel coated PE showed the behavior different from untreated PE. The pendulum with hydrogel coated PE gently attenuated, and the swing of pendulum was maintained for a long time. The attenuation became the gentlest, when hyaluronic acid was used as the lubricant. The friction coefficient became lower value of about 0.01 or below. This result is very similar to the friction in rubbing ceramics ball vs. PVA cup.

\subsection{The water content effect of the gel layer}

Figure 4 shows the relationship between swing frequency and friction coefficient by using the glycerin and hyaluronic

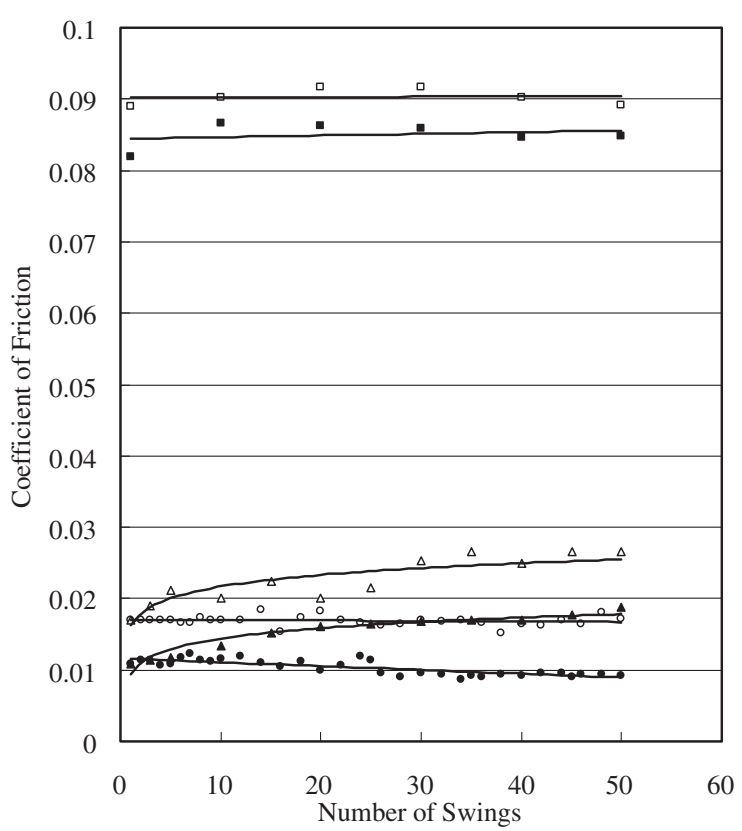

Fig. 4 Effect of water content in the gel layer. Glycerin (open) and hyaluronic acid (close) were used as lubricants. Triangle: Loading weight is not removed during swing course. Circle: Loading weight is during every swing intervals of $3 \mathrm{~min}$. to absorb HA lubricant. Square: The friction coefficient of untreated PE vs. Steel cup Table 1 Coefficient of friction of SUS316/PE using various lubricants. 
Table 1 Frictional coefficient of various lubricants.

\begin{tabular}{cll}
\hline \multirow{1}{*}{ Ball } & Lubricant & Coefficient of friction \\
\hline \multirow{4}{*}{ Untreated PE } & Glycerin & 0.09 \\
\cline { 2 - 3 } & Hyaluronic acid & 0.085 \\
\cline { 2 - 3 } & DMAA homopolymer & 0.1 \\
\cline { 2 - 3 } & Distilled water & 0.1 \\
\cline { 2 - 3 } & silicon oil & 0.1 \\
\cline { 2 - 3 } & non lubricant & 0.1 \\
\hline \multirow{3}{*}{ Hydrogel coated PE } & Glycerin & $0.017 \sim 0.018$ \\
\cline { 2 - 3 } & Hyaluronic acid & $0.009 \sim 0.01$ \\
\cline { 2 - 3 } & Distilled water & $0.04 \sim 0.05$ \\
\cline { 2 - 3 } & silicon oil & $0.35 \sim 0.45$ \\
\cline { 2 - 3 } & non lubricant & 0.4 \\
\hline
\end{tabular}

acid. With decreasing the water from gel layer by the continuous measurement, the friction coefficient of the hydrogel rose gradually. But, when the water was made to soak sufficiently into gel layer by the interval, the friction coefficient of the hydrogel kept the low value.

\subsection{Effect of the lubricant}

Table 1 shows the result of the friction coefficient measured using various lubricants. Friction coefficient of untreated PE was about 0.1 in all lubricants. In the contrary, the friction coefficient of hydrogel coated PE was high value $(0.4)$ in the siloxane and without lubrication.

\subsection{Load property of Hydrogel coated Polyethylene head}

Figure 5 shows extinction curve of the pendulum of each load in HA lubrication. The attenuation of the amplitude was gradually at all load. Figure 6 shows the relationship between friction coefficient and the load. The friction coefficient of the hydrogel coated PE was below 0.01 at all load. This attributed the low frictional coefficient of hydrogel coated $\mathrm{PE}$ to the hydrodynamic-lubrication phenomena.

\subsection{Influence of biological factor}

Figure 7 shows the effect of biological factors, which is included in joints. By the Albumin addition to hyaluronic

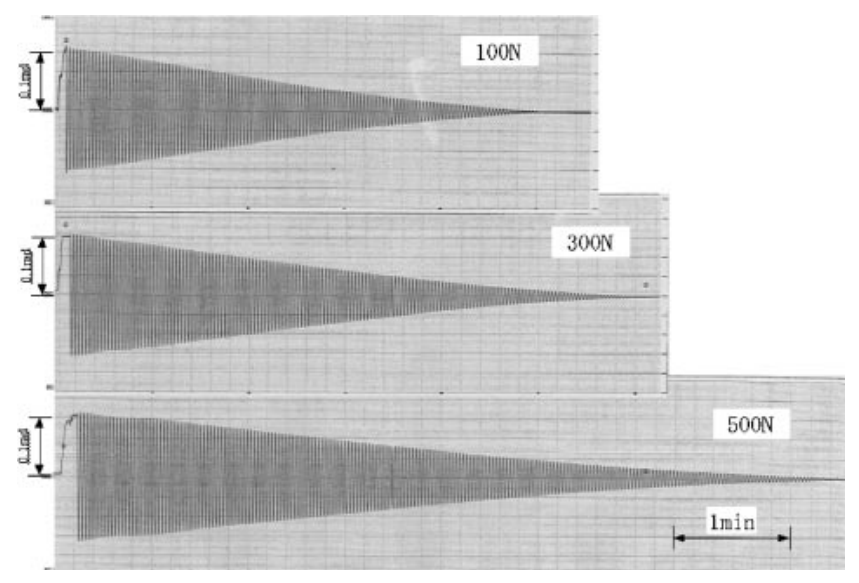

Fig. 5 Damping pattern of a pendulum oscillation.

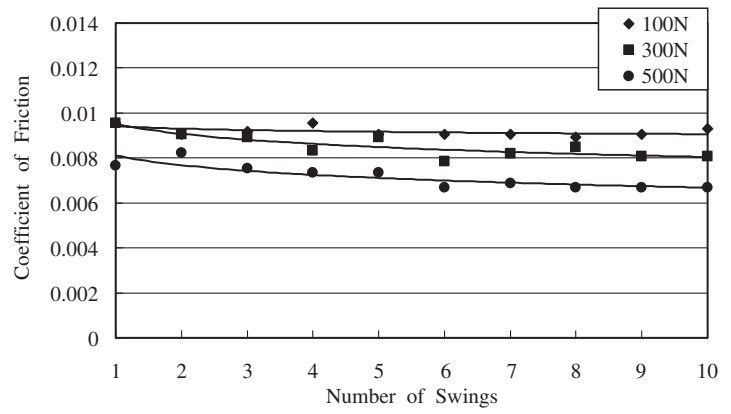

Fig. 6 The effects of load on Hydrogel-coated polyethylene.

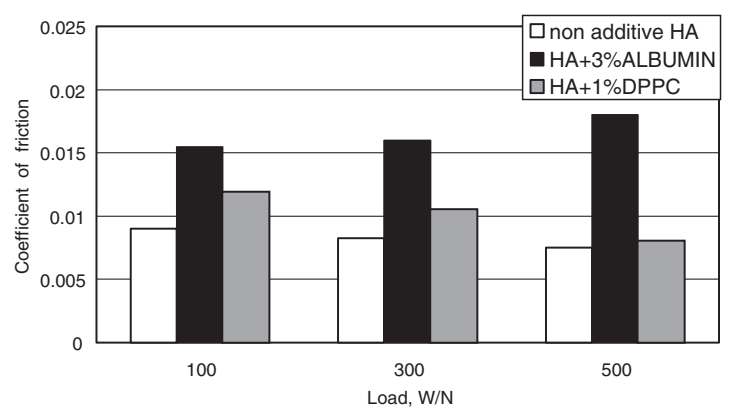

Fig. 7 The effects of biological factor on Hydrogel-coated polyethylene.

acid, the friction coefficient became $0.015 \sim 0.018$, and increased with increasing load. By the DPPC addition to hyaluronic acid, the friction coefficient became $0.008 \sim$ 0.012 . Both lubricants showed a little increase in friction coefficient from plain hyaluronic acid aqueous solution.

\section{Discussion}

We assumed that the low frictional coefficient of hydrogel coated PE was due to the hydrodynamic lubricated phenomena. In brief, we considered that a fluid film was retained by firm liquid restrictive force of the hydrophilic macromolecule chain. In the case of continuous measurement, the friction coefficient rose with increasing in the number of measurement. It was indicated that water content in gel layer influenced the friction coefficient.

Also, it was noticed that increase of the friction coefficient was explained by the following reason: The lubricating liquid in the hydrogel layer was gradually pressed out by applying the load on the hydrogel. The water molecule was attracted by the hydrophilic macromolecule chains and remained in hydrogel layer. Hydrogel with the good hydrodynamic lubrication mechanism was considered to behave like a high viscous fluid, in other words a high-dense colloid gel. Therefore, the friction coefficient of hydrogel was increased with decreasing the water content in hydrogel layer, i.e. with increasing apparent viscosity. This is similar to the phenomenon of the joint. ${ }^{9,10)}$

In the work carried out the pendulum test in the cadaver hip joint (Mabuchi et al.), the friction coefficient was 0.005 after the load. However, the friction coefficient exceeded 0.1 in the load post-30 minute. The friction coefficient increased with the dependence on time course. As the result, friction 
coefficient of hydrogel coated PE was high value (0.4) in the siloxane and unlubricating. We assumed that it was strained without completely binding the siloxane in the hydrophilic macromolecule chain, and polymer chain contacted the cup like the no lubricated condition.

We expected that the increase of the load pressed out the lubricant in gel layer early and the rise in the friction coefficient. However, the friction coefficient lowered with the increase of the load. With the load increase, the concave was formed by the elastic deformation of PE and gel layer. The EHL is promoted by the concave trapping the lubricant. To decrease the friction coefficient, the elastic deformation might have contributed.

Since the albumin and DPPC made chemisorption in the hydrophilic macromolecule chain, we guessed that the waterholding capacity of the gel decreased and the friction coefficient rose.

In order to verify this assumption, hydrogel coated PE was examined by measuring the friction coefficient in following order. (1) HA lubrication, (2) Albumin additive HA lubrication, (3) HA lubrication, after the sample is cleaned in the distilled water by ultrasonic wave. (The result is shown in Fig. 8)

As a result, even if it was sufficiently washed, the friction coefficient, which rose with the albumin addition, did not return in low friction. It was indicated that the albumin made chemisorption in the hydrophilic macromolecule chain.

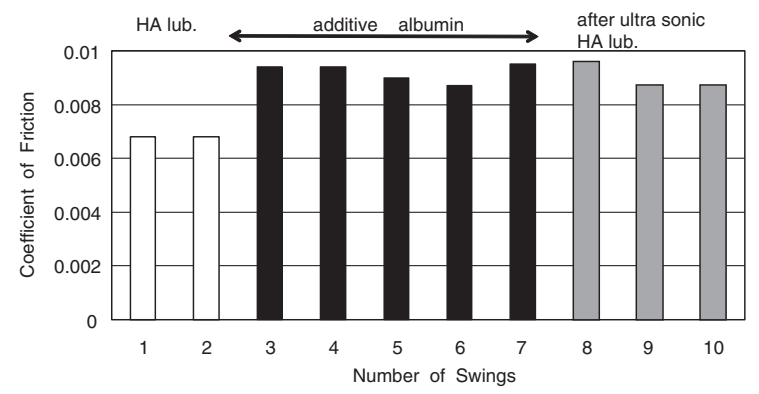

Fig. 8 influence of chemisorption.

\section{Conclusion}

On the result of examining the frictional property of hydrogel coated PE by using pendulum type friction tester, the friction coefficient of the hydrogel was about 0.015 in the glycerin, and about 0.01 in HA. Even the increase in load or biological factor addition, the friction coefficient of the hydrogel is very low.

Hydrogel in which the hydrophilic macromolecule chain sufficiently hydrated showed a low frictional coefficient, which is similar to friction coefficient of the human joint. It is assumed that the EHL can be promoted by covering the hydrogel. In addition, the function is structurally similar to the articular cartilage.

The hydrogel coating is an effective method as clarification of lubrication mechanism of artificial joint and surface modification of artificial joint.

From these experimental facts, we considered that the low friction shown in hydrogel coated PE head is owing to a formation of high viscous zone.

\section{Acknowledgements}

The authors express appreciation to Dr. Sakamoto, assistant professor of Chiba Institute of Technology for his valuable advice on the graft polymerization of hydrogel.

\section{REFERENCES}

1) D. Dowson: Proc. Inst. Mech. Eng. 181 (1967) 45-54.

2) T. Sasada: Journal of Japan Society of lubrication Engineers 23, 2 (1978) 79-84.

3) C. W. McCtuchen: Wear. 5 (1962) 1-17.

4) T. Sasada, Y. Tsukamoto and K. Mabuchi: Biotribology, (sangyou tosyo, 1988).

5) Tadashi Sasada: Proc. 11th Biotribology symposium (1991) 3.

6) Y. Ogiwara, T. Kimura and H. Kubota: J. Appl. Polym. Sci. 27 (1982) 3743-3750.

7) K. Yamada, T. Kitamura, H. Tsutaya and M. Hirata: J. Appl. Polym.Sci. 16 (1992) 1065-1085.

8) K. Ikeuchi, T. Takii, H. Norikane, N. Tomita, T. Ohsumi, Y. Uyama and Y. Ikada: Wear. 161 (1993) 179-185.

9) T. Sasada: Journal of Japanese Society for Biomaterials 3, 3 (1985) 279-285.

10) P. S. Walker: Ann. Rheum. Dis. 27 (1968) 512-520. 